\title{
Significado del cuidado para estudiantes y profesores del Programa de Enfermería de la Universidad Francisco de Paula Santander ${ }^{1}$
}

\author{
Dianne Sofía González Escobarz
}

doi:10.11144/Javeriana.IE17-1.scep

Cómo citar: González Escobar DS. Significado del cuidado para estudiantes y profesores del Programa de Enfermería de la Universidad Francisco de Paula Santander. Investig Enferm. Imagen Desarr. 2015;17(1):77-95. http://dx.doi.org/10.11144/Javeriana. IE17-1.scep

1. Articulo original de investigación. Recibido: 8 de mayo de 2014. Revisado: 15 de junio a 4 de julio de 2014. Aceptado: 24 de julio de 2014.

2. Docente titular, Facultad Ciencias de la Salud, Universidad Francisco de Paula Santander, Cúcuta, Colombia. Enfermera, Pontificia Universidad Javeriana, Bogotá, Colombia. Magíster en Educación de Adultos, Universidad de San Buenaventura, Colombia. Magíster en Cuidado de Enfermaria Paciente Crónico, Universidad Nacional de Colombia. Correo electrónico: dsgonzal@hotmail.es 


\section{Resumen}

Objetivo: El estudio realizado tuvo como objetivo describir el significado del cuidado para estudiantes y profesores del programa de enfermería en el segundo semestre del 2011. Material y métodos: Investigación de tipo descriptivo exploratorio con abordaje cuantitativo, realizado con una muestra conformada por 258 estudiantes y 29 profesores. Para la recolección de información se empleó la Escala de Evaluación del Significado del Cuidar (EESC), escala ordinal tipo Likert con 45 items distribuidos en las cinco categorias de cuidado: característica humana, imperativo moral, afecto, relación interpersonal e intervención terapéutica. El análisis estadístico de la información se basó en el cálculo de medidas descriptivas y pruebas no paramétricas como el test de Kruskal-Wallis (estadística H), coeficiente de concordancia de W de Kendall y prueba de Wilcoxon, todas bajo un nivel de significancia igual a 0,05. Resultados: En los resultados generales de estudiantes y profesores, la categoría con mayor puntuación promedio fue el cuidado como intervención terapéutica, mientras que la categoría con menor puntuación fue el cuidado como afecto. Sin embargo, la percepción del cuidado es diferente entre estudiantes y profesores; se identificaron diferencias estadísticamente significativas entre las categorias: característica humana, afecto e intervención terapéutica, y se observó que la percepción del cuidado frente a estas categorías es más alta en los profesores. Conclusión: Según los resultados no hay consenso cuando se trata del cuidado; hay diferentes percepciones sobre el cuidado que existen simultáneamente y no son excluyentes. Estas formas de percibir el cuidado influyen en la conceptualización y la práctica de enfermería.

Palabras clave: cuidado de enfermeria; significado; estudiantes; profesores

\section{Meaning of Care for Students and Teachers from the Faculty of the Nursing Program at the University Francisco de Paula Santander}

\section{Abstract}

Objective: This study aimed to describe the meaning of care for students and teachers of the nursing program in the second half of 2011. Methods: descriptive exploratory research with a quantitative approach, performed in a sample of 258 students and 29 teachers. To collect information it was used the Assessment Scale for Care Meaning, in Spanish: Escala de Evaluación Del Significado Del Cuidar (EESC, for its intials in spanish), ordinal Likert scale with 45 items divided into five categories: Human characteristic, moral imperative, affection, interpersonal relationship and therapeutic intervention. The statistical analysis of data was based on the calculation of descriptive measures and non-parametric tests such as the Kruskal-Wallis test ( $\mathrm{H}$ statistic), Kendall's coefficient of concordance and Wilcoxon test, all under a significance level equal to 0.05 . Results: In the overall performance of students and teachers, the category with the highest average score was care as therapeutic intervention, while the category with the lowest score was the care and affection. However, perception of care is different between students and teachers; statistically significant differences between categories were identified: human characteristic, affection and therapeutic intervention, and it was found that the perception of care regarding these categories is higher in teachers. Conclusion: We found no consensus when it comes to caring; there are different perceptions of care that exist simultaneously and are not exclusive. These ways of perceiving the care influence the conceptualization and practice of nursing. 


\section{Significado do cuidado em discentes e docentes do Programa de Enfermagem da Universidade Francisco de Paula Santander}

\section{Resumo}

Objetivo: O estudo realizado teve como objetivo descrever o significado do cuidado em discentes e docentes do programa de enfermagem no segundo semestre de 2011. Material e métodos: Pesquisa de tipo descritivo exploratório com abordagem quantitativo, realizado com amostra conformada por 258 alunos e 29 professores. Para a coleta de informação foi usada a Escala de Avaliação do Significado de Cuidar (EASC), escala ordinal tipo Likert com 45 itens distribuídos nas cinco categorias de cuidado: característica humana, imperativo moral, afeto, relação interpessoal e intervenção terapêutica. A análise estadística da informação foi baseada no cálculo de medidas descritivas e testes não-paramétricos como o teste de Kruskal-Wallis (estadística H), coeficiente de concordância de W de Kendall e proba de Wilcoxon, tudo sob um nível de significância igual a 0,05. Resultados: Nos resultados gerais de alunos e professores, a categoria com maior pontuação média foi o cuidado como intervenção terapêutica, enquanto que a categoria com menor pontuação foi o cuidado como afeto. No entanto, a percepção do cuidado é diferente entre alunos e professores; identificaram-se diferencias estatisticamente significativas entre as categorias: característica humana, afeto e intervenção terapêutica, e observou-se que a percepção do cuidado face a estas categorias é mais alta nos professores. Conclusão: Segundo os resultados não tem consenso quando trata do cuidado; tem diferentes percepções sobre o cuidado que se dão simultaneamente e não são excludentes. Estas formas de perceber o cuidado influenciam na conceptualização e a prática da enfermagem.

Palavras-chave: cuidado de enfermagem; significado; discentes; docentes 


\section{Introducción}

En la actualidad, el cuidado es reconocido como algo esencial para la vida - no exclusivo de enfermería- e inherente a las relaciones humanas; sin embargo, el proceso de conceptualización ha sido históricamente difícil, por no tratarse de un valor social y económicamente dominante.

El cuidado ha sido objeto de estudio y reflexión filosófica. De acuerdo con Reich (1), la palabra cuidar tiene al menos cuatro aceptaciones: 1) significa ansiedad, preocupación, angustia o sufrimiento mental; 2) puede significar preocupación o interés básico por las personas, las instituciones o las ideas que tienen importancia para uno mismo; 3) es la atención solicita y responsable ante el trabajo, y 4) implica tener respeto y atender las necesidades específicas de una persona en particular.

El universo del cuidado comprende categorías del ámbito ontológico, antropológico, epistemológico y pedagógico. Esas categorías envuelven todo cuanto rodea y responde a la esencia del cuidado: ser una persona cuidada, las obligaciones incluidas en el cuidado, lo que hace la persona cuando cuida, cómo el cuidado es conocido, es aprendido y es enseñado. El cuidado, más que un acto, es una actitud, y abarca más que un momento de atención, de ocupación, es una preocupación (2).

Para Zea (3), cuidar como objeto de estudio ha adquirido a lo largo de los años una estructura conceptual que alcanza cada vez mayor valor y significado en el entorno social; pero lo más relevante del cuidado como arte y disciplina es la institucionalización dentro de la enfermería como su esencia; es decir, el cuidado se ha instaurado en ella como norte, como objetivo central de la atención. Se cuidan comunidades e individuos y se les provee condiciones para ello; así se les hace partícipes activos tanto en su autocuidado como en la educación que puedan recibir. Es el cuidar la esencia de la profesión de enfermería que, por su naturaleza, se brinda al otro o a uno mismo, bajo condiciones de salud vulneradas o de riesgo, un cuidado digno, que propicia sentimientos de interacción y reciprocidad propios del ser humano.

Para los seguidores del cuidado humano, el cuidar es entendido como la esencia del ser. Si el cuidar es lo que caracteriza las acciones de enfermería en sus diferentes dimensiones, y es parte del ser cuidador, todas sus acciones deben inscribirse en el cuidado. Ese cuidado significa realizar acciones acompañadas, siempre, de actitudes y comportamientos de cuidar (4).

Compartiendo los planteamientos de Kuerten y cols. (5), el concepto de cuidado se construye permanentemente a partir del conocimiento científico técnico del profesional, de la cultura, valores y creencias tanto del profesional como de la persona cuidada y su cuidador, influenciados por el contexto específico en el que se desarrolla la experiencia de cuidado.

Morse y cols. (6) encontraron 35 definiciones del concepto de cuidado, entre las cuales identifican cinco líneas de significado básicas en la conceptualización ontológica de cuidado. Producto del análisis de múltiples estudios, logran identificar si el autor consideraba el cuidado un rasgo humano, parte de la naturaleza humana o característica básica del ser humano; si era visto como imperativo moral; como una emoción y un 
sentimiento de compasión; un compromiso mutuo entre la enfermera y el paciente como una interacción, o si era percibido como una intervención terapéutica. De las definiciones implícitas y explícitas del cuidado identificadas, surge la clasificación ontológica del cuidado como característica o rasgo humano, ideal o imperativo moral, afecto, relación interpersonal y acción o intervención terapéutica.

La significación de un objeto es lo que este representa para la práctica social y depende de las funciones que desempeñe en la actividad de las personas. El sentido de los significados está determinado por factores objetivos de la realidad y el razonamiento, o bien por factores subjetivos como deseos, tendencias, objetivos e intenciones sociales y personales de los sujetos.

El significado que se tiene de un fenómeno condiciona la forma en que es representada la realidad en los sujetos. Estas representaciones se estructuran, según Daza y cols. (7), alrededor de tres componentes fundamentales: la actitud hacia el objeto, la información sobre el objeto y un campo de representación donde se organizan jerárquicamente una serie de contenidos.

En enfermería, el significado está ligado al sentido que las acciones tienen para quienes las realizan: cuanto más abstracto es el concepto, más compleja y variable es la identificación de sus atributos. Los significados se modifican y estabilizan mediante los usos y procesos de interacción social que se dan en las diferentes situaciones o experiencias.

Con el proceso formativo, el estudiante inicia el desarrollo de competencias profesionales como la autonomía, el razonamiento crítico, el compromiso social y habilidades técnicas específicas, asî como un sistema de valores que le permiten entender al otro, adoptar una postura transformadora y de compromiso con el desarrollo y crecimiento de la disciplina y la profesión. De manera simultánea, en el acercamiento a la práctica profesional - a través de las experiencias prácticas formativas, en el encuentro con otros profesionales, estudiantes, profesores y sujetos de cuidado- van construyendo su identidad profesional y consolidando su estructura conceptual respecto al cuidado, la enfermería y la persona.

Estudios acerca del significado del cuidado para el profesional de enfermería evidencian la diversidad existente, así como el cuidado homologado a la atención oportuna, rápida, continua y permanente, orientada a la resolución de problemas particulares que afectan a las personas que demandan un servicio. Por otra parte, se define como un conjunto de categorías que involucran la comunicación verbal y no verbal, minimización del dolor físico, empatía para atender el todo y el envolvimiento que alude a la relación cercana entre el cuidador y el ser cuidado como finalidad terapéutica $(8,9)$.

El objetivo de esta investigación fue describir el significado que tiene el cuidado para estudiantes y profesores del Programa de Enfermería de la Universidad Francisco de Paula Santander.

Explorar el significado que tiene el cuidado para los estudiantes y profesores del Programa de Enfermería permitirá conocer qué entienden 
por cuidado y la forma como lo abordan en la práctica. Los profesores, en su práctica profesional como enfermeros(as)-formadores, y los estudiantes en las prácticas formativas en las que desarrollan sus competencias y construyen su identidad profesional.

El conocimiento sobre los conceptos de cuidado permitirán al programa examinar de manera reflexiva el proceso y resultados del enfoque dado a los componentes curriculares y las estrategias pedagógicas empleadas para alcanzar los propósitos de formación y comprender actitudes, conocimientos y actuaciones de sus estudiantes, docentes y egresados en el desarrollo de la práctica profesional.

\section{Metodología}

El presente estudio corresponde a una investigación de tipo descriptivo exploratorio, de corte transversal con abordaje cuantitativo. Se aplicó un muestreo no probabilístico y la muestra quedó conformada por 258 estudiantes y 29 profesores, que aceptaron participar del estudio. La recolección de la información se llevó a cabo de forma individual y personal durante el segundo semestre del 2011, con apoyo de estudiantes del semillero de investigación del Grupo de Cuidado.

Para la realización del estudio se empleó la Escala de Evaluación del Significado del Cuidar (EESC), instrumento diseñado y validado por la doctora Rosa Aparecida Pavan Bison, al cual se le añadieron unas preguntas de información general que permitieron delinear el perfil de la población participante (10). Este instrumento contiene una escala ordinal tipo Likert, con 45 ítems distribuidos en las 5 categorías de cuidado: característica humana, imperativo moral, afecto, relación interpersonal e intervención terapéutica con 9 afirmaciones en cada una de ellas. Cada item fue calificado según la percepción de los estudiantes y profesores de 1 a 5 , donde 1 representa total desacuerdo con la afirmación, y 5, total acuerdo. En cada categoría se puede obtener un puntaje entre 9 y 45 puntos: a mayor puntaje obtenido, mayor correspondencia con la concepción de cuidado que representa la categoría. A escala general la puntuación está entre 45 y 225. De la puntuación total se obtiene un valor medio por categoría de cuidado y por cada grupo estudiado.

El análisis estadístico de la información se basó en el cálculo de medidas descriptivas, distribuciones simples de frecuencias y la realización de pruebas no paramétricas como el test de Kruskal-Wallis (estadística $\mathrm{H}$ ) para comparación de grupos, coeficiente de concordancia de W de Kendall y la prueba de Wilcoxon para la comparación de las categorías de cuidado dentro del mismo grupo, todas bajo un nivel de significancia igual a 0,05.

En el desarrollo del estudio se aplicaron los principios éticos de la investigación, contando para la recolección de la información con el consentimiento informado de los participantes. Se atendió lo establecido en la Resolución 008430 de 1993, que determina las normas científicas, técnicas y administrativas para la investigación en salud. 


\section{Resultados y análisis}

\section{Características sociodemográficas}

Respecto a la caracterización sociodemográfica de los estudiantes, se encontró que el 81,4\% era mujer, con rango de edad predominante de 15 a 20 años (68,2\%), seguido del de 21 a 25 años (27,1\%) y 26 a 30 años $(4,7 \%)$. E1 95,7\% era soltero, depende económicamente de sus padres el 85,3\%; de otro familiar, el 5,4\%, y de su pareja, un 4,3\%.

Estos resultados coinciden con otros estudios en los que se realiza una caracterización sociodemográfica de estudiantes de enfermería en el país. Los aspectos en los que se encuentran mayor similitud son proporción de hombres y mujeres, predominio del rango de edad que ubica a la población estudiantil en la etapa de la adolescencia, condiciones socioeconómicas, estado civil y dependencia económica $(11,12)$.

De los profesores participantes, el $31 \%$ corresponde a profesores de tiempo completo del programa, y el 69\%, a contratación de hora cátedra. La edad promedio en el grupo fue de 43,7 \pm 8,6 años; la edad de los profesores varía entre los 24 y los 55 años; el grupo de edad más representativo es el de 40 a 44 años. Del total de profesores entrevistados, solo uno hace referencia al género masculino. El 65,5\% de los(as) profesores estaban casados o vivían en unión libre; el 27,6\% era separado o soltero, y el 6,9\% es viudo.

E1 $13,8 \%$ de los(as) docentes lleva menos de 10 años de haber egresado de la universidad; el 34,5\%, entre 10 y 19 años; el 41,4\%, entre 20 y 29 años, y el 10,3\%, 30 años o más de haber egresado. El comportamiento frente al tiempo de estar trabajando es similar al de haber egresado, es decir, los(as) profesores empezaron su actividad laboral inmediatamente después del egreso de la universidad. Con respecto a las áreas de desempeño, prevalecen el área clínica asistencial, con el 79,3\%, y el área administrativa, con el 24,1\%. Además de la docencia, el 41,4\% labora actualmente en la parte clínica; un 10,3\%, en salud pública, y un 6,9\%, en el área administrativa. En cuanto a los estudios de posgrado, sobresalen gerencia y auditoría de la calidad (31\%), especialización en pedagogía (20,7\%) y maestría en enfermería (17,2\%). E1 $31 \%$ de los profesores refirió no haber realizado ninguna especialización.

Los resultados obtenidos son similares a los hallazgos de Rozo y Abaunza (13), en las variables sociodemográficas y laborales de los profesionales docentes de los programas de enfermería del nororiente colombiano. El estudio reporta predominio del género femenino con un 91,5\%. Un $60,6 \%$ de las docentes están casadas o en unión libre; entre tanto, el $28,7 \%$ corresponde a las solteras. Respecto al nivel de estudios, la mayoría de los docentes de los programas cuentan con especialización como preparación académica (64,9\%), seguido de maestría (20,2\%). En cuanto a la forma de vinculación, la mayor parte de la población $(46,8 \%)$ la tiene por hora cátedra. 
Por otra parte, Pavan y cols. (10), en su estudio sobre el significado de cuidar entre estudiantes y profesionales de enfermería que incluye estudiantes de pregrado y especialización, profesionales de servicio y profesores, reportan características sociodemográficas que difieren de resultados obtenidos en este trabajo en lo que respecta a media de edad y estado civil. No establece una relación entre estas características y la percepción de significado de cuidado en los diferentes grupos.

\section{Significado de cuidar}

En cuanto a las puntuaciones en las categorias de cuidado, se encuentra correspondencia entre el grupo de profesores y estudiantes, de las cuales la categoría con mayor puntuación promedio es el cuidado como intervención terapéutica, con 41,6 \pm 3 puntos y $40 \pm 4$ puntos, respectivamente. De igual modo, la categoría con menor puntuación promedio en ambos grupos fue el cuidado como afecto, con $37,9 \pm 3,9$ puntos y $36 \pm 4$ puntos.

En relación con la escala general, la puntuación promedio en el grupo de estudiantes fue igual a 191,2 \pm 16 puntos; mientras en los profesores fue igual a 199,5 \pm 13,2 puntos. Las figuras 1 y 2 muestran los diagramas de caja respectivos para los resultados de la evaluación en ambos grupos.

La percepción del cuidado varía entre estudiantes y profesores ( $p$ $=0,01)$. Se identificaron diferencias estadísticamente significativas entre las categorías: característica humana $(p=0,00)$, afecto $(p=0,01)$ e intervención terapéutica $(\mathrm{p}=0,03)$, y se observó que la percepción del cuidado frente a estas categorías es más alta por parte de los profesores (tabla 1).

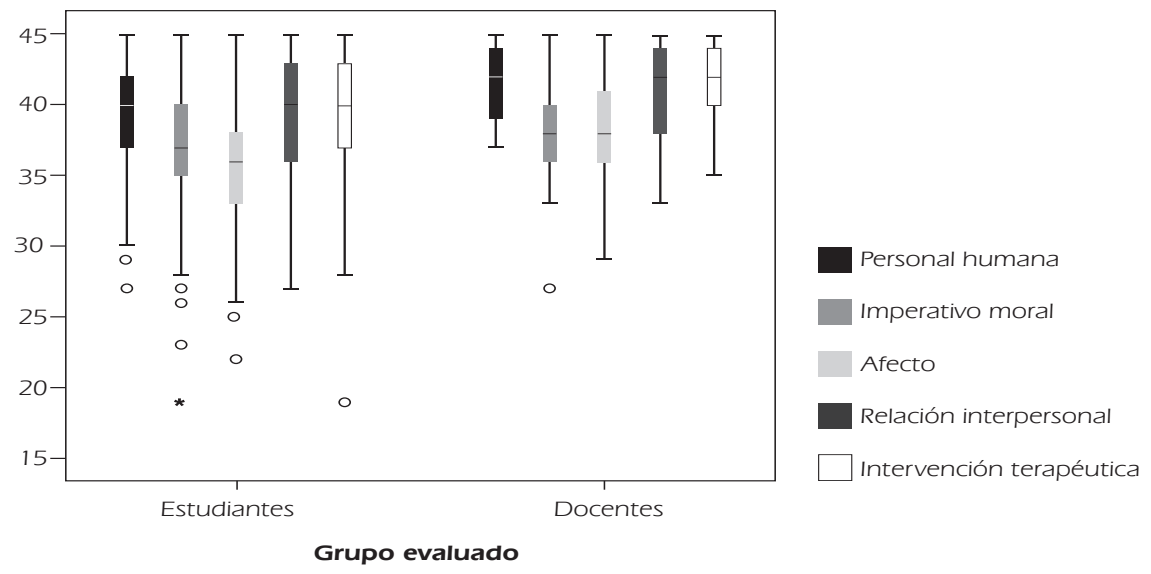

Figura 1. Diagrama de caja por categoría del cuidado en cada grupo evaluado 


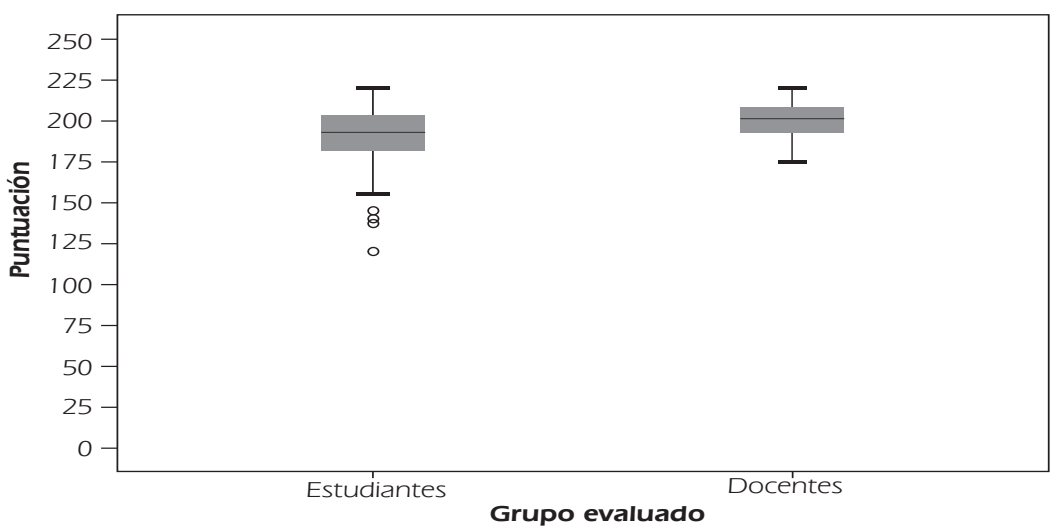

Figura 2. Diagrama de caja para la escala de percepción del cuidado según el grupo evaluado

Fuente: resultados escala de evaluación significado de cuidado aplicada a estudiantes y profesores del Programa de Enfermería, 2011.

Tabla 1. Test de Kruskal-Wallis para la comparación de medianas en resultados por categoría entre los grupos evaluados

\begin{tabular}{|c|c|c|c|c|c|c|}
\hline $\begin{array}{c}\text { Categoria del } \\
\text { cuidado }\end{array}$ & $\begin{array}{l}\text { Grupo eva- } \\
\text { luado }\end{array}$ & $\mathbf{N}$ & Rango promedio & $\begin{array}{c}\text { Chi } \\
\text { cuadrado } \\
\text { (Kruskal- } \\
\text { Wallis) } \\
\end{array}$ & g1 & $\begin{array}{l}\text { Nivel de } \\
\text { significa } \\
\text { ción }\end{array}$ \\
\hline \multirow{3}{*}{$\begin{array}{l}\text { Característica } \\
\text { humana }\end{array}$} & Estudiantes & 265 & 142,3 & \multirow{3}{*}{10,1} & \multirow{3}{*}{1} & \multirow{3}{*}{0,00} \\
\hline & Docentes & 29 & 195,0 & & & \\
\hline & Total & 294 & & & & \\
\hline \multirow{3}{*}{$\begin{array}{l}\text { Imperativo } \\
\text { moral }\end{array}$} & Estudiantes & 265 & 145,7 & \multirow{3}{*}{1,2} & \multirow{3}{*}{1} & \multirow{3}{*}{0,27} \\
\hline & Docentes & 29 & 164,1 & & & \\
\hline & Total & 294 & & & & \\
\hline \multirow{3}{*}{ Afecto } & Estudiantes & 265 & 143,3 & \multirow{3}{*}{6,5} & \multirow{3}{*}{1} & \multirow{3}{*}{0,01} \\
\hline & Docentes & 29 & 185,5 & & & \\
\hline & Total & 294 & & & & \\
\hline \multirow{3}{*}{$\begin{array}{l}\text { Relación inter- } \\
\text { personal }\end{array}$} & Estudiantes & 265 & 144,5 & \multirow{3}{*}{3,4} & \multirow{3}{*}{1} & \multirow{3}{*}{0,06} \\
\hline & Docentes & 29 & 175,2 & & & \\
\hline & Total & 294 & & & & \\
\hline \multirow{3}{*}{$\begin{array}{l}\text { Intervención } \\
\text { terapéutica }\end{array}$} & Estudiantes & 265 & 143,9 & \multirow{3}{*}{4,8} & \multirow{3}{*}{1} & \multirow{3}{*}{0,03} \\
\hline & Docentes & 29 & 180,3 & & & \\
\hline & Total & 294 & & & & \\
\hline \multirow{3}{*}{ Total } & Estudiantes & 265 & 143,18 & \multirow{3}{*}{4,8} & \multirow{3}{*}{1} & \multirow{3}{*}{0,01} \\
\hline & Docentes & 29 & 186,97 & & & \\
\hline & Total & 294 & & & & \\
\hline
\end{tabular}

Fuente: resultados de la escala de evaluación significado de cuidado aplicada a estudiantes y profesores del Programa de Enfermería, 2011. 
Al evaluar el grado de concordancia intragrupos frente a la percepción del cuidado y sus categorías (tabla 2), se logró concluir que los resultados sobre percepción del cuidado son diferentes entre categorías, tanto para alumnos como para los profesores $(p=0,00)$.

TABla 2. Coeficiente de concordancia de Kendall para los resultados de percepción de cuidado por categorías, intragrupos

\begin{tabular}{|c|c|c|c|c|c|c|}
\hline \multicolumn{7}{|c|}{ Estudiantes } \\
\hline \multirow[b]{2}{*}{$\begin{array}{l}\text { Categoria del } \\
\text { cuidado }\end{array}$} & \multirow[b]{2}{*}{$\begin{array}{c}\text { Rango } \\
\text { promedio }\end{array}$} & \multicolumn{5}{|c|}{ Estadisticos de contraste } \\
\hline & & $\mathbf{N}$ & $\begin{array}{c}\text { W de } \\
\text { Kendall }\end{array}$ & $\begin{array}{l}\text { Chi cua- } \\
\text { drado }\end{array}$ & g1 & $\begin{array}{l}\text { Nivel de } \\
\text { significa- } \\
\text { ción }\end{array}$ \\
\hline $\begin{array}{l}\text { Característica } \\
\text { humana }\end{array}$ & 3,44 & \multirow{5}{*}{265} & \multirow{5}{*}{0,3} & \multirow{5}{*}{317,7} & \multirow{5}{*}{4} & \multirow{5}{*}{0,00} \\
\hline $\begin{array}{l}\text { Imperativo } \\
\text { moral }\end{array}$ & 2,43 & & & & & \\
\hline Afecto & 1,82 & & & & & \\
\hline $\begin{array}{l}\text { Relación inter- } \\
\text { personal }\end{array}$ & 3,49 & & & & & \\
\hline \begin{tabular}{|l|}
$\begin{array}{l}\text { Intervención } \\
\text { terapéutica }\end{array}$ \\
\end{tabular} & 3,82 & & & & & \\
\hline \multicolumn{7}{|c|}{ Docentes } \\
\hline \multirow[b]{2}{*}{$\begin{array}{l}\text { Categoria del } \\
\text { cuidado }\end{array}$} & \multirow[b]{2}{*}{$\begin{array}{c}\text { Rango } \\
\text { promedio }\end{array}$} & \multicolumn{5}{|c|}{ Estadísticos de contraste } \\
\hline & & $\mathbf{N}$ & $\begin{array}{c}\text { W de } \\
\text { Kendall }\end{array}$ & $\begin{array}{l}\text { Chi cua- } \\
\text { drado }\end{array}$ & gl & $\begin{array}{l}\text { Nivel de } \\
\text { significa- } \\
\text { ción }\end{array}$ \\
\hline $\begin{array}{l}\text { Característica } \\
\text { humana }\end{array}$ & 3,88 & \multirow{5}{*}{29} & \multirow{5}{*}{0,4} & \multirow{5}{*}{44,4} & \multirow{5}{*}{4} & \multirow{5}{*}{0,00} \\
\hline $\begin{array}{l}\text { Imperativo } \\
\text { moral }\end{array}$ & 2,02 & & & & & \\
\hline Afecto & 1,97 & & & & & \\
\hline $\begin{array}{l}\text { Relación inter- } \\
\text { personal }\end{array}$ & 3,33 & & & & & \\
\hline $\begin{array}{l}\text { Intervención } \\
\text { terapéutica }\end{array}$ & 3,81 & & & & & \\
\hline
\end{tabular}

Fuente: resultados de la escala de evaluación significado de cuidado aplicada a estudiantes y profesores del Programa de Enfermería, 2011.

En el grupo de estudiantes, la percepción del cuidado como intervención terapéutica prevalece sobre las demás categorías $(p=0,00)$. La percepción de cuidado como característica humana predomina de manera significativa sobre categorías como el afecto e imperativo moral $(p=0,00)$; frente al cuidado como relación interpersonal la percepción es semejante $(p=0,65)$.

La percepción del cuidado como relación interpersonal destaca sobre las de afecto e imperativo moral $(\mathrm{p}=0,00)$. La percepción del cuidado como imperativo moral es significativamente mayor que la percepción del cuidado como afecto $(\mathrm{p}=0,00)$ (tabla 3$)$. 
TABla 3. Prueba de signos de Wilcoxon para comparar resultados por categorias en los estudiantes

\begin{tabular}{|c|c|c|c|c|c|c|}
\hline \multicolumn{7}{|c|}{ Comparación intragrupos: estudiantes } \\
\hline \multicolumn{2}{|c|}{ Pares de comparaciones } & \multirow{2}{*}{$\begin{array}{c}\mathbf{N} \\
180 \\
\end{array}$} & \multirow{2}{*}{\begin{tabular}{|r|}
$\begin{array}{c}\text { Rango } \\
\text { promedio }\end{array}$ \\
138,4 \\
\end{tabular}} & \multirow{2}{*}{\begin{tabular}{|c|}
$\begin{array}{c}\text { Suma de } \\
\text { rangos }\end{array}$ \\
24915,0 \\
\end{tabular}} & $\mathbf{Z}$ & $\begin{array}{l}\text { Nivel de sig- } \\
\text { nificación }\end{array}$ \\
\hline \multirow{4}{*}{$\begin{array}{l}\text { Imperativo moral- } \\
\text { característica } \\
\text { humana }\end{array}$} & Rangos negativos & & & & \multirow{4}{*}{$-7,62$} & \multirow{4}{*}{0,00} \\
\hline & Rangos positivos & 73 & 98,8 & 7216,0 & & \\
\hline & Empates & 12 & & & & \\
\hline & Total & 265 & & & & \\
\hline \multirow{4}{*}{$\begin{array}{l}\text { Afecto- } \\
\text { característica } \\
\text { humana }\end{array}$} & Rangos negativos & 208 & 135,2 & 28121,0 & \multirow{4}{*}{$-10,88$} & \multirow{4}{*}{0,00} \\
\hline & Rangos positivos & 42 & 77,5 & 3254,0 & & \\
\hline & Empates & 15 & & & & \\
\hline & Total & 265 & & & & \\
\hline \multirow{4}{*}{$\begin{array}{l}\text { Relación } \\
\text { interpersonal- } \\
\text { característica } \\
\text { humana }\end{array}$} & Rangos negativos & 108 & 112,6 & 12164,0 & \multirow{4}{*}{$-0,45$} & \multirow{4}{*}{0,65} \\
\hline & Rangos positivos & 116 & 112,4 & 13036,0 & & \\
\hline & Empates & 41 & & & & \\
\hline & Total & 265 & & & & \\
\hline \multirow{4}{*}{$\begin{array}{l}\text { Intervención } \\
\text { terapéutica- } \\
\text { caracteristica } \\
\text { humana }\end{array}$} & Rangos negativos & 99 & 99,7 & 9872,0 & \multirow{4}{*}{$-3,30$} & \multirow{4}{*}{0,00} \\
\hline & Rangos positivos & 130 & 126,6 & 16463,0 & & \\
\hline & Empates & 36 & & & & \\
\hline & Total & 265 & & & & \\
\hline \multirow{4}{*}{$\begin{array}{l}\text { Afecto-imperativo } \\
\text { moral }\end{array}$} & Rangos negativos & 152 & 123,2 & 18725,5 & \multirow{4}{*}{$-4,96$} & \multirow{4}{*}{0,00} \\
\hline & Rangos positivos & 81 & 105,4 & 8535,5 & & \\
\hline & Empates & 32 & & & & \\
\hline & Total & 265 & & & & \\
\hline \multirow{4}{*}{$\begin{array}{l}\text { Relación interper- } \\
\text { sonal-imperativo } \\
\text { moral }\end{array}$} & Rangos negativos & 64 & 86,6 & 5541,5 & \multirow{4}{*}{$-8,43$} & \multirow{4}{*}{0,00} \\
\hline & Rangos positivos & 178 & 134,1 & 23861,5 & & \\
\hline & Empates & 23 & & & & \\
\hline & Total & 265 & & & & \\
\hline & Rangos negativos & 46 & 77,8 & 3579,5 & \multirow{4}{*}{$-10,23$} & \multirow{4}{*}{0,00} \\
\hline $\begin{array}{l}\text { Intervención } \\
\text { terapéutica-impe- }\end{array}$ & Rangos positivos & 196 & 131,8 & 25823,5 & & \\
\hline $\begin{array}{l}\text { terapeutica-1mpe- } \\
\text { rativo moral }\end{array}$ & Empates & 23 & & & & \\
\hline & Total & 265 & & & & \\
\hline & Rangos negativos & 31 & 95,4 & 2957,0 & & \\
\hline Relación interper- & Rangos positivos & 221 & 130,9 & 28921,0 & -1123 & \\
\hline sonal-afecto & Empates & 13 & & & $-11,23$ & 0,00 \\
\hline & Total & 265 & & & & \\
\hline & Rangos negativos & 23 & 68,6 & 1578,5 & & \\
\hline Intervención & Rangos positivos & 223 & 129,2 & 28802,5 & & \\
\hline terapéutica-afecto & Empates & 19 & & & ,20 & 0,00 \\
\hline & Total & 265 & & & & \\
\hline & Rangos negativos & 78 & 96,9 & 7557,5 & & \\
\hline Intervención tera- & Rangos positivos & 129 & 108,3 & 13970,5 & & \\
\hline péutica-relación & Empates & 58 & & & $-3,74$ & 0,00 \\
\hline interpersonal & Total & 265 & & & & \\
\hline
\end{tabular}

Fuente: resultados de la escala de evaluación significado de cuidado aplicada a estudiantes y profesores del Programa de Enfermería, 2011. 
En cuanto a los profesores, la percepción de cuidado como intervención terapéutica predomina ante categorías como el afecto e imperativo moral $(\mathrm{p}=0,00)$; frente a las categorias característica humana y relación interpersonal, no existen diferencias significativas $(\mathrm{p}=0,94 \mathrm{y} \mathrm{p}=0,15$, respectivamente).

La percepción de cuidado como característica personal humana prevalece sobre las categorías afecto e imperativo moral $(\mathrm{p}=0,00)$; la percepción de cuidado por parte de los profesores como característica humana y como relación interpersonal es semejante $(\mathrm{p}=0,11)$.

Al igual que en los estudiantes, la percepción del cuidado de los profesores como relación interpersonal sobresale de manera estadísticamente significativa sobre las categorías afecto e imperativo moral ( $p=0,00)$. En los profesores no se identifican diferencias estadisticas entre las categorias afecto e Imperativo moral $(\mathrm{p}=0,99)$ (tabla 4).

Con el análisis de los resultados obtenidos mediante la aplicación de la ESSC se pueden identificar diferencias y similitudes en la percepción del significado del cuidado en el grupo de profesores y estudiantes en las categorías exploradas. Esos resultados muestran que no hay consenso cuando se trata del cuidado; existen simultáneamente diferentes percepciones sobre el cuidado y no son excluyentes. Estas formas de percibir el cuidado influyen en la conceptualización y la práctica, y al tiempo que se desarrollan y explican afectan el avance y relevancia de la acción de enfermería.

Existe predominio de la percepción de cuidado como intervención terapéutica en profesores y estudiantes. En esta concepción del cuidado, según lo plantea Morse, citado por Salazar y Martínez (14), la enfermera debe detectar y satisfacer las carencias manifiestas del paciente, campo donde se deben destacar las competencias y habilidades con relación al cuidado que promueven la autonomía, el autocuidado, la información, la compasión y la promoción de la vida. Hace hincapié en la necesidad de adecuar el conocimiento y las habilidades como base de las acciones de cuidado; también, como la congruencia entre las acciones de enfermería y la percepción de las necesidades del paciente.

La interpretación del cuidado como intervención terapéutica frente a un problema de salud de las personas en términos de respuestas medibles se relaciona con la influencia recibida desde la perspectiva paradigmática en enfermería denominada particular determinística. Históricamente la práctica de enfermería ha estado enraizada en la provisión de un cuidado físico bajo el control médico; ha formado la base del conocimiento y la práctica de la enfermería y ha promovido la percepción del paciente como un cuerpo biológico que es atendido por la enfermera (15). La influencia de esta visión de la realidad conduce a conceptualizaciones que se traducen en elementos concretos de la práctica del cuidado, el proceso formativo, la indagación y construcción del conocimiento.

Daza y Medina (8), desde la experiencia docente, han encontrado que durante la formación se pone el relieve en la dimensión técnico-científica y en la dimensión relacional. Sin embargo, al explorar la realidad de 
la práctica profesional cotidiana se percibe que la dimensión técnico-científica se magnifica en detrimento de la relacional, que queda relegada a un segundo plano por diversos factores de índole administrativo y burocrático que necesitan ser estudiados.

TABla 4. Prueba de signos de Wilcoxon para comparar resultados por categorías en los docentes

\begin{tabular}{|c|c|c|c|c|c|c|}
\hline \multicolumn{7}{|c|}{ Comparación intragrupos: docentes } \\
\hline \multicolumn{2}{|c|}{ Pares de comparaciones } & $\mathbf{N}$ & $\begin{array}{c}\text { Rango } \\
\text { promedio }\end{array}$ & $\begin{array}{l}\text { Suma de } \\
\text { rangos }\end{array}$ & $\mathbf{Z}$ & $\begin{array}{c}\text { Nivel de } \\
\text { significación } \\
\text { (bilateral) }\end{array}$ \\
\hline \multirow{4}{*}{$\begin{array}{l}\text { Imperativo moral- } \\
\text { personal humana }\end{array}$} & Rangos negativos & 23 & 15,07 & 346,50 & \multirow{4}{*}{$-3,79$} & \multirow{4}{*}{0,00} \\
\hline & Rangos positivos & 4 & 7,88 & 31,50 & & \\
\hline & Empates & 2 & & & & \\
\hline & Total & 29 & & & & \\
\hline \multirow{4}{*}{$\begin{array}{l}\text { Afecto-personal } \\
\text { humana }\end{array}$} & Rangos negativos & 24 & 13,33 & 320,00 & \multirow{4}{*}{$-4,25$} & \multirow{4}{*}{0,00} \\
\hline & Rangos positivos & 1 & 5,00 & 5,00 & & \\
\hline & Empates & 4 & & & & \\
\hline & Total & 29 & & & & \\
\hline \multirow{4}{*}{$\begin{array}{l}\text { Relación } \\
\text { interpersonal- } \\
\text { personal humana }\end{array}$} & Rangos negativos & 15 & 12,70 & 190,50 & \multirow{4}{*}{$-1,61$} & \multirow{4}{*}{0,11} \\
\hline & Rangos positivos & 8 & 10,69 & 85,50 & & \\
\hline & Empates & 6 & & & & \\
\hline & Total & 29 & & & & \\
\hline \multirow{4}{*}{$\begin{array}{l}\text { Intervención } \\
\text { terapéutica- } \\
\text { personal humana }\end{array}$} & Rangos negativos & 13 & 11,73 & 152,50 & \multirow{4}{*}{$-0,07$} & \multirow{4}{*}{0,94} \\
\hline & Rangos positivos & 11 & 13,41 & 147,50 & & \\
\hline & Empates & 5 & & & & \\
\hline & Total & 29 & & & & \\
\hline \multirow{4}{*}{$\begin{array}{l}\text { Afecto-imperativo } \\
\text { moral }\end{array}$} & Rangos negativos & 14 & 15,50 & 217,00 & \multirow{4}{*}{$-0,01$} & \multirow{4}{*}{0,99} \\
\hline & Rangos positivos & 15 & 14,53 & 218,00 & & \\
\hline & Empates & 0 & & & & \\
\hline & Total & 29 & & & & \\
\hline \multirow{4}{*}{$\begin{array}{l}\text { Relación } \\
\text { interpersonal- } \\
\text { imperativo moral }\end{array}$} & Rangos negativos & 4 & 11,50 & 46,00 & \multirow{4}{*}{$-3,45$} & \multirow{4}{*}{0,00} \\
\hline & Rangos positivos & 23 & 14,43 & 332,00 & & \\
\hline & Empates & 2 & & & & \\
\hline & Total & 29 & & & & \\
\hline \multirow{4}{*}{$\begin{array}{l}\text { Intervención } \\
\text { terapéutica- } \\
\text { imperativo moral }\end{array}$} & Rangos negativos & 5 & 6,50 & 32,50 & \multirow{4}{*}{$-3,89$} & \multirow{4}{*}{0,00} \\
\hline & Rangos positivos & 23 & 16,24 & 373,50 & & \\
\hline & Empates & 1 & & & & \\
\hline & Total & 29 & & & & \\
\hline
\end{tabular}

Fuente: resultados de la escala de evaluación significado de cuidado aplicada a estudiantes y profesores del Programa de Enfermería, 2011. 
Desde estos referentes es comprensible que predomine el significado de cuidado como acción o intervención terapéutica entre estudiantes y profesores del programa; este resultado coincide con estudios realizados en otros países de América Latina, donde las mayores frecuencias encontradas se refieren al cuidado como intervención terapéutica, lo cual indica la profesionalización del cuidado en el nivel de su significación conceptual $(10,16)$. Las interacciones terapéuticas forman un conjunto de acciones profesionales que tienen como objetivos el mantenimiento de la salud del ser humano, el restablecimiento del equilibrio, el alivio del dolor y la cura de los males. Teniendo por base los presupuestos humanistas, el profesor de enfermería, conductor del proceso educativo, privilegia la enseñanza de los conceptos y técnicas para alcanzar estos objetivos.

Asimismo, aunque no se encuentran en la literatura colombiana suficientes referencias de estudios orientados a la identificación de la percepción del cuidado en estudiantes de enfermería, el trabajo realizado en la Universidad Nacional de Colombia que describe la percepción de cuidado con base en los patrones del conocimiento de enfermeria muestra que los comportamientos de cuidado relacionados con el patrón empírico son los que obtienen la puntación más importante en un $65,8 \%$ de los participantes. Esta situación que no debe generar sorpresa, puesto que se accede al conocimiento a través de la descripción, explicación y predicción de los eventos de la práctica y relacionados con las habilidades clínicas (12).

El significado del cuidado como relación interpersonal o interacción personal comparte la mayor puntuación con la categoría intervención terapéutica en el grupo de profesores y en el de estudiantes con la categoría cuidado como característica humana. La interacción se entiende como el intercambio de sentimientos, pensamientos, acciones y experiencias entre la enfermera y el paciente, en la cual se ejerce una influencia recíproca con el objeto de lograr una intervención terapéutica de calidad. Chacón y Giraldo, citados por Daza (8), consideran que es una dimensión básica en el cuidado de enfermería, porque al centrarse en el contacto entre personas, se constituye en móvil indispensable para llevarlo a cabo.

Para Salazar y Martínez (14), en el cuidado como relación interpersonal o interacción personal hay comunicación, confianza, respeto y compromiso de uno con el otro. Cuando esto ocurre, ambos se enriquecen. La integración de los sentimientos compartidos establece la posibilidad de dar o recibir ayuda. Se hace hincapié en la integración de sentimientos y acciones en el encuentro, de tal manera que la interacción enfermerapaciente es cualitativamente diferente a un encuentro sin cuidado. Desde esta perspectiva, se cree que la interacción enfermera-paciente es la esencia del cuidado y abarca sentimientos y comportamientos que ocurren en la relación.

En la relación enfermera-paciente confluyen percepciones, sentimientos, actitudes y conocimientos que caracterizan los comportamientos de los participantes de la interacción; cada uno percibe y responde como persona. En el caso de la enfermera, también como profesional, con acciones de cuidado en respuesta a las necesidades percibidas en el sujeto de 
cuidado. De esta manera, puede explicarse la identificación del significado de cuidar de los profesores del programa como cuidado terapéutico y relación interpersonal con un mismo grado de valoración.

El cuidado como característica humana es la segunda categoría con mayor puntaje en el grupo de estudiantes y comparte la mayor puntuación en el grupo de docentes con el significado como intervención terapéutica y relación interpersonal. El cuidado como característica humana es visto como innato, o sea, todos los seres poseen el potencial del cuidado; sin embargo, la habilidad de cuidar no es uniforme. Como motivadora de sus acciones, la enfermería posee esta característica innata, pero sus diferencias en la práctica de la forma de cuidar reflejan las diferencias en los niveles de entendimiento del significado salud-enfermedad (5).

Arenas (2) plantea que hablar del cuidado como condición humana es pensar y hablar cómo el cuidado es vivido, cómo se siente. No tenemos cuidado, somos todo cuidado. Arenas (2), al explicar el cuidado como característica humana cita a Boff, quien refiere que esto significa que posee una dimensión ontológica que entra en la constitución del ser humano. Es un modo de ser singular de hombres y mujeres. Sin cuidado dejamos de ser humanos. El cuidado es siempre preocupación y solicitud, confiere sentido a la existencia humana.

En el grupo de profesores, la percepción del cuidado como característica humana obtuvo el mismo puntaje que las categorías relación interpersonal e intervención terapéutica. Sin embargo, se evidencia afectación en las declaraciones que guardan relación con la percepción de autocuidado como atención a la propia salud, actividad física, ocio y alimentación adecuada, pues no hacen parte de los elementos representativos que integran el significado de cuidado de este grupo. Aspectos que coinciden con lo encontrado por Lázaro y cols., citado en Hernández y cols. (16), donde las afirmaciones relacionadas con la apariencia del cuidador tuvieron un índice de desempeño general óptimo. Contrariamente, en los aspectos estudiados en que era sugerida la interiorización perceptiva del cuidado consigo mismo, se pudo observar que la atención con la propia salud, confort, alimentación y actividad física no era tan prioritaria como significación del cuidado.

Al analizar los resultados en el grupo de estudiantes se encuentra que la mayoría de las afirmaciones relacionadas con esta categoría tuvo un comportamiento homogéneo en sus medianas; sin embargo, es evidente la tendencia a no considerar prioritario lo relacionado con el propio cuidado, específicamente respecto a una adecuada alimentación, actividad física y ocio. Estos resultados corresponden con otro estudio donde se exploran las prácticas de autocuidado y de riesgo de estudiantes de enfermería, en el cual se encuentra que los estudiantes presentan déficit de autocuidado en cuanto a los hábitos alimenticios, la eliminación y medidas de higiene (17).

La percepción de cuidado como imperativo moral en el grupo de estudiantes y profesores se encuentra en un mismo nivel por los puntajes obtenidos respecto a las otras categorías. En ambos grupos esta percep- 
ción es menor a la de cuidado como intervención terapéutica, característica humana y relación interpersonal.

En el grupo de profesores y estudiantes se encuentra tendencia al desacuerdo en las afirmaciones que valoran esta categoría que plantean: "ejercer la enfermería es una tarea difícil" y "cuidar es un acto de compasión". En las restantes, la opinión de los participantes se encuentra entre de acuerdo y totalmente de acuerdo, con mayor puntuación en el grupo de profesores. Es contradictorio que a pesar de considerar que el cuidado no es acto de compasión, reconocen que implica compromiso de un ser humano con otro; implica servicio, responsabilidad, respeto, honestidad, relación de confianza y solidaridad.

Garzón (18), al referirse a los aspectos éticos del acto de cuidado, plantea que un elemento fundamental en el cuidado es la relación enfermera-paciente, que se construye sobre la ética del respeto al otro como interlocutor válido, y la intersubjetividad y la comunicación efectiva teniendo en cuenta y respetando los aspectos culturales, los valores y las creencias. En los diferentes comportamientos, conductas e intervenciones del profesional de enfermería y de su equipo, al realizar el acto de cuidado se debe traducir la dimensión filosófica, ética y bioética, de humanización y científico-técnica que fundamenta su conocimiento y su actuar, y que la sociedad percibe y valora como buen cuidado.

La práctica del cuidado se caracteriza por una fuerte dimensión ética, debido a las decisiones que involucra en pro de preservar la dignidad e integridad de las personas que necesitan de tratamiento y cuidado profesional. Todas las decisiones de enfermería corresponden a una dimensión moral, en el sentido de que sus actuaciones y juicios involucran la existencia de otros seres humanos.

El cuidado como afecto es la categoría con el promedio más bajo entre las categorías evaluadas en los grupos estudio. Revisando la valoración dada a las afirmaciones que representan esta dimensión del cuidado, se identifica una tendencia a la supresión y control de los sentimientos en el acto de cuidado. En el grupo de profesores y estudiantes se manifiesta duda respecto a si la enfermera debe expresar sus sentimientos durante el cuidado, y en si la dulzura debe estar presente o no. Por otra parte, se muestran de acuerdo en que siendo impersonal, la enfermera(o) cuida mejor.

Para Quintero (19), el afecto se traduce en comportamientos y actitudes. Todo sentimiento tiene sus motivaciones, intereses y propósitos. Es el motor o el inhibidor de la acción de cuidar, pues moviliza o inhibe a la persona. Desde una perspectiva profesional, tenemos muchas fortalezas clínicas en el momento de la valoración. El soporte de datos objetivos y subjetivos nos permite actuar. Sin embargo, en ocasiones el abordaje de dimensiones como espiritualidad y afecto se diluyen en el hacer. La enfermería ha de centrar su quehacer en la perspectiva del afecto y la ternura cuidando intuitiva y amorosamente a otros. Por tanto, el cuidado como una forma de amar, posibilita la expresión de sentimientos y emociones 9) traducidos en un hacer. 
De acuerdo con Watson, referido por Rivera y Triana (20), la identificación de los sentimientos conduce a la actualización y autoaceptación de la enfermera y del paciente. Las enfermeras, al reconocer su sensibilidad y sus sentimientos, se vuelven más genuinas, auténticas y sensibles hacia los demás. La enfermera tiene que estar preparada para los sentimientos positivos y negativos, reconociendo que la comprensión intelectual y la comprensión emocional de una situación son diferentes.

Es habitual que en la práctica sanitaria se separe el desarrollo profesional de este ámbito, por lo cual las personas tienden a esconder, negar o disimular sus sentimientos. Esto llevará a no tratar con los propios sentimientos, a temerles y a no poder cambiarlos cuando estos no son positivos. Un individuo que rehúsa reconocer sus propios sentimientos, difícilmente logrará entender los de los otros. Por otra parte, se tiende a proteger al paciente de sentimientos negativos, para que no llore o muestre ansiedad. Estos sentimientos forman parte de la vida y de los procesos que vivimos como seres humanos (21).

\section{Conclusiones}

La percepción de cuidado en profesores y estudiantes como intervención terapéutica, característica humana y relación interpersonal son componentes de la representación del significado de cuidado. En el marco de la relación docente-estudiante existente, el predominio de estas categorías pone en evidencia el papel que tiene la educación en el desarrollo de actitudes, conductas y valores, incluidos aspectos técnico-científicos, interpersonales, afectivos y éticos relacionados con el cuidado.

En profesores y estudiantes se evidencia afectación en el reconocimiento del cuidar como acto individual que se da a sí mismo. Las declaraciones que guardan relación con la percepción de autocuidado como atención con la propia salud, actividad física, ocio y alimentación adecuada no hacen parte de los elementos representativos que integran el significado de cuidado en los grupos. Así mismo, la poca valoración del cuidado como imperativo moral y afecto respecto a las otras categorias revela la necesidad de reflexión sobre las estrategias de formación empleadas para el abordaje de dimensión ética, afectiva y humanizada del cuidado en el programa, puesto que el cuidar profesional puede ser aprendido, pero la compasión y la solidaridad, al igual que otros valores, es algo que solo se reconoce a través de las experiencias.

\section{Recomendaciones}

Dar continuidad al estudio de esta temática con enfoque cualitativo, de tal forma que sea posible identificar otros significados, asî como la existencia de cambios a través del proceso formativo.

\section{Conflicto de intereses}

En el desarrollo del proyecto de investigación y proceso de publicación de los resultados no se presentó ningún tipo de conflicto de intereses. La financiación del proyecto fue con recursos propios del investigador. 


\section{Referencias}

1. Reich WT. Contemporany ethics of care. En: Reich W, editor. Encyclopedia of bioethics. 2nd ed. Vol. 1. New York: Simon \& Shuste/ MacMillan; 1996.

2. Arenas NM. El cuidado como manifestación de la condición humana. En: Salus Online [internet]. 2006 [citado 2011 jun 13];10(1):1927. Disponible en: http://salus-online.fcs.uc.edu.ve/cuidado_manifestacion_humana.pdf.

3. Zea LE. Cuidar de otros: condición humana y esencia de una profesión. Investigación y Educación en Enfermería. 2003;21(2):154-8.

4. Waldow R. Enseñanza de enfermería centrada en el cuidado. Aquichan. 2009;9(3):246-56.

5. Kuerten P, Do Prado ML, De Gasperi P, Sebold LF, Waterkemper R, C. Bub MB. El cuidado y la enfermería. Av Enferm [internet]. 2009 Jul [citado 2012 jun 15];27(1):102-9. Disponible en: http:// www.scielo.org.co/scielo.php?script=sci_arttext\&pid=S012145002009000100011\&lng=en.

6. Morse J, Solberg SM, Neander WL, Bottorff JL, Johnson JL. Concepts of caring and caring as a concept. ANS Adv Nurs Sci. 1990;13(1):1-14.

7. Daza R, Torres AM, Prieto GI. Análisis crítico del cuidado de enfermería: interacción, participación y afecto. Index Enferm [internet]. 2005 Jun [citado 2012 abr 24];14(48-49):18-22. Disponible en: http://scielo.isciii.es/scielo.php?script=sci_arttext\&pid=S1132$12962005000100004 \& 1 n g=$ es. http://dx.doi.org/10.4321/S113212962005000100004

8. Daza R, Medina LS. Significado del cuidado en enfermería desde la perspectiva de los profesionales de una institución hospitalaria de tercer nivel en Santa Fe de Bogotá, Colombia. Cultura de los Cuidados. 2006;19(10):55-62.

9. Baggio MA. O significado de cuidado para profissionais da equipe de enfermagem. Rev Electr Enf [internet]. 2006 [citado 2012 ene 5];8(1):9-16. Disponible en: http://www.fen.ufg.br/revista/revista8_1/original_01.htm.

10. Pavan R, Ferreira AR, Ferreira JL. La percepción del cuidar entre estudiantes y profesionales de enfermería. Index Enferm [internet]. 2007 Jun [citado 2012 may 29]; 16(56): 30-4. Disponible en: http://scielo.isciii.es/scielo.php?script=sci_arttext\&pid=S1132$12962007000100007 \& \operatorname{lng}=$ es.

11. Rodríguez M, Pineda SA, Vélez LF. Características del consumo de tabaco en estudiantes de enfermería de la Universidad de Antioquia (Colombia). Invest Educ Enferm [internet]. 2010 Nov [citado 2012 jun 12];28(3):370-83. Disponible en: http://www.scielo.org.co/scielo. php?script $=$ sci_arttext\&pid=S0120-53072010000300008\&lng=en.

12. Gómez OJ, Daza LA, Zambrano VM. Percepción de cuidado en estudiantes de enfermería: caracterización e impacto para la formación y la visión del ejercicio profesional. Av Enferm [internet]. 2008 Jul [citado 2012 jun 12];26(1):85-96. Disponible en: http:// 
www.scielo.org.co/scielo.php?script=sci_arttext\&pid=S012145002008000100009\&lng=es.

13. Rozo SM, Abaunza M. Liderazgo transaccional y transformacional. Av Enferm [internet]. 2010 Dec [citado 2012 jun 12];28(2):62-72. Disponible en: http://www.scielo.org.co/scielo.php?script=sci_ arttext\&pid=S0121-45002010000200006\&lng=en.

14. Salazar AM, Martínez C. Un sobrevuelo por algunas teorías donde la interacción enfermera-paciente es el núcleo del cuidado. Av Enferm [internet]. 2008 [citado 2012 abr 3]; [aprox. 12 p.]. Disponible en: http://www.revistas.unal.edu.co/index.php/avenferm/article/ view/12945/13664.

15. Durán M. Marco epistemológico de la enfermería. Aquichan [internet]. 2009 [citado 2012 jun 25];2(1):7-18. Disponible en: http://aquichan.unisabana.edu.co/index.php/aquichan/article/view/17/35.

16. Hernández LL, Zequeira DM, Miranda A. La percepción del cuidado en profesionales de enfermería. Cubana Enfermer [internet]. 2010 [citado 2012 oct 15];26(1):30-41. Disponible en: http://scielo.sld.cu/ scielo.php?script=sci_arttext\&pid=S0864-03192010000100007.

17. Giraldo A, León DM, Navarro YL, Trejos O. Determinación de prácticas de autocuidado y prácticas de riesgo en los patrones nutricional y de eliminación en un grupo de estudiantes del Programa de Enfermería de la Universidad de Caldas. Hacia Promoc Salud [internet]. 2002 ene-dic [citado 201229 jun]; [aprox. 11 p.]. Disponible en: http:// promocionsalud.ucaldas.edu.co/downloads/Revista\%207_13.pdf.

18. Garzón N. Ética profesional y teorías de enfermería. Aquichán [internet]. 2005 [citado 2012 nov 6]; [aprox. 9 p.]. Disponible en: http://aquichan.unisabana.edu.co/index.php/aquichan/article/view/60/125.

19. Quintero MC. Espiritualidad y afecto en el cuidado de enfermería. En: Grupo de Cuidado: cuidado y práctica de enfermería. Bogotá: Universidad Nacional de Colombia; 2006. p. 184-91.

20. Rivera LN, Triana Á. Cuidado humanizado de enfermería: visibilizando la teoría y la investigación en la práctica, en la Clínica del Country. Av Enferm [internet]. 2007 [citado 2012 jun 20];10(4):1521. Disponible en: http://www.slideshare.net/karenmelissadx/kfc2.

21. Urra E. Jana A, García M. Algunos aspectos esenciales del pensamiento de Jean Watson y su teoría de cuidados transpersonales. Cienc Enferm [internet]. 2011 Dic [citado 2012 nov 9 ];17(3):11-22. Disponible en http://www.scielo.cl/scielo.php?script=sci_arttext\&pid=S071795532011000300002\&lng=es\&nrm=iso. 